\title{
FRECUENCIA DE HALLAZGOS DE VARIANTES ANATÓMICAS DE LOS SENOS MAXILARES EN TOMOGRAFÍAS COMPUTARIZADAS DE HAZ CÓNICO: ESTUDIO PILOTO
}

\section{FREQUENCY OF ANATOMIC VARIATIONS IN MAXILLARY SINUS WITH THE AID OF CONE BEAM COMPUTED TOMOGRAPHY(CBCT): PILOT STUDY}

\author{
Gutiérrez Mesa Manuela $^{1 *}$, Ruiz-García de Chacón Vilma ${ }^{2}$ León Manco Roberto $^{3}$ \\ ${ }^{1}$ Residente de segundo año de la segunda especialidad en Radiología Oral y Máxilofacial de la Universidad Peruana \\ Cayetano Heredia. Perú. \\ 2 Departamento Académico de Medicina y Cirugía Bucomáxilofacial de la Universidad Peruana Cayetano Heredia. Perú. \\ ${ }^{3}$ Departamento Académico de Odontología Social de la Universidad Peruana Cayetano Heredia. Perú. \\ *manuela.gutierrez.m@upch.pe
}

\begin{abstract}
Resumen
Resumen: Los senos paranasales son cavidades dentro de los huesos de la cara y el cráneo, están relacionados directamente con las fosas nasales su función es el calentamiento del aire que entra a través de las fosas nasales, participan en la fonación, la respiración y la percepción del olfato, al igual que las demás estructuras dentro del macizo facial pueden presentar variantes anatómicas consideradas como normales y que no están relacionadas con algún tipo de patología o el inicio de las mismas. El objetivo del presente estudio piloto fue evaluar las variantes anatómicas de los senos maxilares mediante tomografía cone beam. Se revisaron 40 tomografías con beam, para un total de 80 senos maxilares y se evaluaron las siguientes variantes anatómicas: agenesia, hipoplasia, exostosis, pseudoseptum, neumatización. Se encontró que 16 senos maxilares (37.2\%) pertenecían al sexo masculino y 27 (62.8\%) pertenecían al sexo femenino. La edad promedio de los pacientes fue 43.7 años con una D.E. de 14.0. En donde $55(68.8 \%)$ presentaron variantes anatómicas. La variante anatómica más frecuente fue la neumatización con un total de 23 casos $(41.8 \%)$, seguida del pseudoseptum con un total de 12 casos (21.8\%), además, se presentaron 8 casos $(14.6 \%)$ de exostosis.
\end{abstract}

Palabras clave: Tomografía Computarizada de Haz Cónico, seno maxilar, huesos faciales .

\begin{abstract}
Summary: Paranasal sinuses are cavities within the bones of the face and skull, they are directly related to the nostrils and their function is the heating of the air that enters during the breath, also, participate in the phonation, breathing and the perception of the smell. Like other structures within the facial mass, they can present anatomical variants considered as normal and that are not related to some type of pathology or the beginning of the same ones. The objective of the present pilot study of the anatomical variants of the maxillary sinuses by cone beam tomography was to evaluate 40 beam scans were reviewed for a total of 80 maxillary sinuses. The following anatomical variants were evaluated: agenesis, hypoplasia, exostosis, pseudoseptum, pneumatization. It was found that 16 maxillary sinuses $(37.2 \%)$ were male and $27(62.8 \%)$ were female. The mean age of the patients was 43.7 years with a S.D. 14.0 years. Where, 55 (68.8\%) presented anatomical variants. The most frequent anatomic variant was pneumatization with a total of 23 cases (41.8\%), followed by a case with a total of 12 cases (21.8\%). In addition, there were 8 cases (14.6\%) of exostosis.
\end{abstract}

Key words: Cone-Beam Computed Tomography, maxillary sinus, facial bones. 


\section{INTRODUCCIÓN}

Los senos paranasales son cavidades dentro de los huesos de la cara y el cráneo. Están relacionados directamente con las fosas nasales y su función tiene que ver principalmente con el calentamiento del aire que entra a través de las fosas nasales. Además, participan en la fonación, la respiración y la percepción del olfato. Los senos paranasales son cuatro y cada uno lleva el nombre del hueso en el que se encuentra: seno frontal, seno etmoidal, seno esfenoidal y los senos maxilares. ${ }^{1}$

Los senos maxilares (SM) se encuentran relacionados directamente con el proceso dentoalveolar, además de que son los de mayor tamaño y se encuentran bilateralmente en los huesos maxilares superiores. ${ }^{2}$ Son dos cavidades dentro del hueso maxilar superior, tienen una forma de pirámide irregular, donde la base se encuentra dirigida hacia la fosa nasal y su vértice hacia el hueso malar. Limita hacia arriba con el borde inferior de la órbita y hacia abajo con las raíces de los dientes posteriores. En su interior están recubiertos por mucosa respiratoria ciliada y secretora de moco que se encarga de atrapar y eliminar cuerpos extraños que ingresan durante la inhalación. Ambos senos maxilares desembocan en el meato medio a través del ostium. ${ }^{3}$ Como cualquiera de los otros senos paranasales pueden presentar variaciones anatómicas de lo normal, tales como: neumatización alveolar uni o bilateral, hipoplasia, agenesia, septum antral y exostosis. Además, pueden presentar patologías como: opacidad, ocupamiento, pseudoquistes de retención mucosa, pólipos, antrolitos y engrosamiento de la mucosa. ${ }^{4,5}$

Conocer las posibles variaciones anatómicas que se pueden hallar dentro de los senos maxilares, es de ayuda al clínico al momento de tomar decisiones en cuanto a diagnóstico, pronóstico y en el desarrollo del plan de tratamiento de los pacientes. ${ }^{6,7}$ El objetivo de este estudio piloto fue determinar la frecuencia de hallazgos de variantes anatómicas de los senos maxilares en Tomografía Computarizada Cone Beam (TCCB) de pacientes que acudieron al Servicio de Radiología Oral y Maxilofacial, Clínica Dental Docente, Universidad Peruana Cayetano Heredia, San Isidro, Lima-Perú, 2016.

\section{MATERIALES Y MÉTODOS}

El presente estudio piloto fue descriptivo, retrospectivo y transversal. Se evaluó 40 TCCB de pacientes que acudieron al Servicio de Radiología Oral y Maxilofacial, Clínica Dental Docente, Universidad Peruana Cayetano Heredia, San Isidro, Lima-Perú, 2016, haciendo un total de 80 SM. Para evaluar la presencia de neumatización se trabajó con la metodología propuesta por Sánchez y colaboradores. ${ }^{8}$ Para la determinación de la presencia de las demás variantes, se realizó una calibración previa con un radiólogo oral y máxilofacial con más de 5 años de experiencia (gold estándar) obteniéndose un valor de Kappa de 0.8. Las imágenes se obtuvieron en un equipo Galileos marca Sirona modelo ComfortPLUS .Se analizaron las imágenes por medio del software Galaxis en una pantalla de 16 pulgadas de marca Lenovo. Cada TCCB, se valoró en los tres planos (axial, coronal y sagital) en la función proyección de máxima intensidad (MIP). Se anotaron las observaciones en las fichas de recolección de elaboradas de forma específica para esta investigación, en donde se consignaron los hallazgos previamente codificados.

Las variables de estudio fueron variantes anatómicas del seno maxilar y tomografía computarizada cone beam. Las variantes anatómicas del seno maxilar se categorizaron en exostosis, septum, pseudoseptum, neumatización e hipoplasia; y las combinaciones posibles de estas categorías, obteniendo finalmente 12 categorías. Posterior a ello, se procedió a un análisis descriptivo de las variables mediante la obtención de frecuencias absolutas y relativas de las variables cualitativas, y el promedio y desviación estándar de las variables cuantitativas. El estudio contó con un nivel de confianza de 955 y un $\mathrm{p}<0.05$. El programa estadístico empleado fue el SPSS v. 24.0.

Se mantuvo el anonimato de los pacientes mediante su codificación y se contó con las autorizaciones del Comité Institucional de Ética de la Universidad Peruana Cayetano Heredia siendo aprobado el 02 de 12 de 2016, con código SIDISI № 100271 .

\section{RESULTADOS}

De los 80 SM evaluados, 16 (37.2\%) pertenecían al sexo masculino y $27(62.8 \%)$ pertenecían al sexo femenino. La edad promedio de los pacientes fue 43.7 con una D.E. de 14.0 (Tabla 1)

La tabla 2 muestra las variantes anatómicas de los SM según lado, sexo y edad en las TCCB de pacientes que acudieron al Servicio de Radiología Oral y Maxilofacial Clínica Dental Docente, Universidad Peruana Cayetano Heredia, San Isidro, Lima-Perú, 2016. De los 80 SM analizados, $55(68.8 \%)$ presentaron variantes anatómicas. De estos, 20 $(25 \%)$ pertenecieron al sexo masculino, mientras que 35 $(43.8 \%)$ pertenecieron al sexo femenino. La edad promedio de los pacientes que presentaron variantes anatómicas del seno maxilar fue de 44.5 años (DE 13.8). La variante anatómica más frecuente fue la neumatización (Fig 1) con un total de 23 casos (41.8\%), seguida del pseudoseptum (Fig 2)

con un total de 12 casos $(21.8 \%)$. Siguiente a estos se hallaron 8 casos $(14.6 \%)$ de exostosis (Fig 1). Luego se encontró 3 casos (5.4\%) de septum (Fig 3). No se presentó ningún caso de hipoplasia pura. Se identificaron las siguientes combinaciones de variantes anatómicas con un caso $(1.8 \%)$ en cada una: hipoplasia + septum, hipoplasia + pseudoseptum, exostosis + septum, exostosis + pseudoseptum, septum + neumatización, pseudoseptum + neumatización, exostosis + pseudoseptum + neumatización. 


\begin{tabular}{|c|c|c|c|c|c|c|c|c|c|c|}
\hline \multirow{3}{*}{ Variante } & \multicolumn{4}{|c|}{ Lado } & \multicolumn{4}{|c|}{ Sexo } & \multirow{2}{*}{\multicolumn{2}{|c|}{ Edad }} \\
\hline & \multicolumn{2}{|c|}{ Derecho } & \multicolumn{2}{|c|}{ Izquierdo } & \multicolumn{2}{|c|}{ Masculino } & \multicolumn{2}{|c|}{ Femenino } & & \\
\hline & n & $\%$ & $\mathbf{n}$ & $\%$ & $\mathbf{n}$ & $\%$ & $\mathbf{n}$ & $\%$ & $\mathrm{x}$ & $\mathrm{DE}$ \\
\hline \multicolumn{11}{|l|}{ Frecuencia } \\
\hline Sin variante & 14 & 17,5 & 11 & 13,8 & 9 & 11,3 & 16 & 20,0 & 42,0 & 14,7 \\
\hline Con variante & 26 & 32,5 & 29 & 36,3 & 20 & 25,0 & 35 & 43,8 & 44,5 & 13,8 \\
\hline \multicolumn{11}{|l|}{ Tipo } \\
\hline Exostosis & 3 & 5,6 & 5 & 9,2 & 2 & 3,7 & 6 & 10,9 & 43,3 & 16,0 \\
\hline Septum & 2 & 3,6 & 1 & 1,8 & 0 & 0,0 & 3 & 5,6 & 56,0 & 6,6 \\
\hline Pseuooseptum & 6 & 10,9 & 6 & 10,9 & 2 & 3,6 & 10 & 18,2 & 38,5 & 12,0 \\
\hline Neumatización & 11 & 20,0 & 12 & 21,8 & 10 & 18,2 & 13 & 23,6 & 46,2 & 14,6 \\
\hline Hipoloasia + Septum & 1 & 1,8 & 0 & 0,0 & 1 & 1,8 & 0 & 0,0 & 34,0 & - \\
\hline Hipoloasia + Pseudoseptum & 0 & 0,0 & 1 & 1,8 & 1 & 1,8 & 0 & 0,0 & 34,0 & - \\
\hline Exostosis + Septum & 0 & 0,0 & 1 & 1,8 & 1 & 1,8 & 0 & 0,0 & 54,0 & - \\
\hline Exostosis + Pseudoseptum & 0 & 0,0 & 1 & 1,8 & 0 & 0,0 & 1 & 1,8 & 46,0 & - \\
\hline Septum + Neumatización & 1 & 1,8 & 1 & 1,8 & 1 & 1,8 & 1 & 1,8 & 62,5 & 0,7 \\
\hline Pseuooseptum + Neumatización & 1 & 1,8 & 1 & 1,8 & 2 & 3,6 & 0 & 0,0 & 34,0 & 0,0 \\
\hline $\begin{array}{l}\text { Exostosis + Pseudoseptum + } \\
\text { Neumatización }\end{array}$ & 1 & 1,8 & 0 & 0,0 & 0 & 0,0 & 1 & 1,8 & 46,0 & - \\
\hline
\end{tabular}

n: Frecuencia absoluta.

$\%$ : Frecuencia relativa.
$\mathrm{X}$ : Promedio

DE: Desviaión estándar.

Tabla 1. Variantes anatómicas de los senos maxilares según lado,sexo y edad en las Tomografias computarizadas Cone Bean

\begin{tabular}{llrrrr}
\hline & Recuento & $\begin{array}{c}\text { \% de N } \\
\text { tablas }\end{array}$ & Media & $\begin{array}{c}\text { Desviación } \\
\text { estándar }\end{array}$ \\
\hline LADO & Derecho & 40 & 50,0 & & \\
& Izquierdo & 40 & 50,0 & & \\
SEXO & Masculino & 16 & 37,2 & & \\
& Femenino & 27 & 62,8 & & \multirow{2}{*}{4,0} \\
EDAD & & & & 43,7 & 14,0
\end{tabular}

Tabla 2. Datos demográficos de los participantes

\section{DISCUSIÓN}

El conocimiento de la anatomía normal de los senos maxilares permite identificar cuando podríamos estar ante una patología, o cuando simplemente estamos frente a una variante de lo normal; por esto, Shiki K y colaboradores ${ }^{9}$ hicieron un estudio que tuvo dos propósitos: primero evaluar las variaciones del seno maxilar en pacientes que esperaban tener implantes dentales y el segundo fue elucidar las limitaciones de la radiografía panorámica para evaluar las variaciones del seno maxilar, así como las tasas de prevalencia de variaciones y lesiones en el seno maxilar en pacientes con implantes

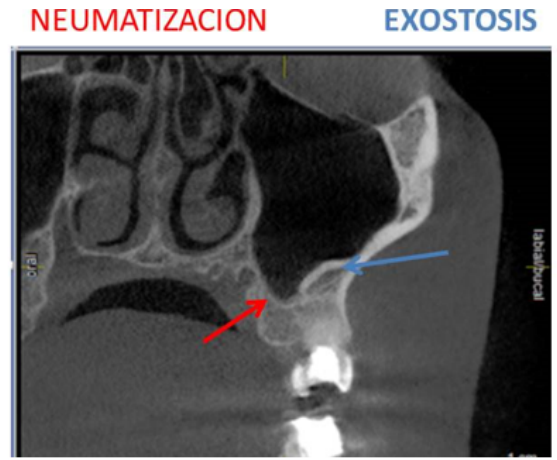

Fig. 1. Neumatización y exostosis de seno maxilar

maxilares usando TCCB. En los resultados se encontraron las siguientes condiciones: neumatización, septum, hipoplasia, aplasia, engrosamiento de la mucosa, quistes de retención y quistes, discontinuidad del piso del seno, retención de fluidos, engrosamiento del hueso, antrolitos, exostosis, opacificación sinusal y cuerpos extraños. Además de que la mayoría de ellas estaban en el grupo de los pacientes con implantes. La 


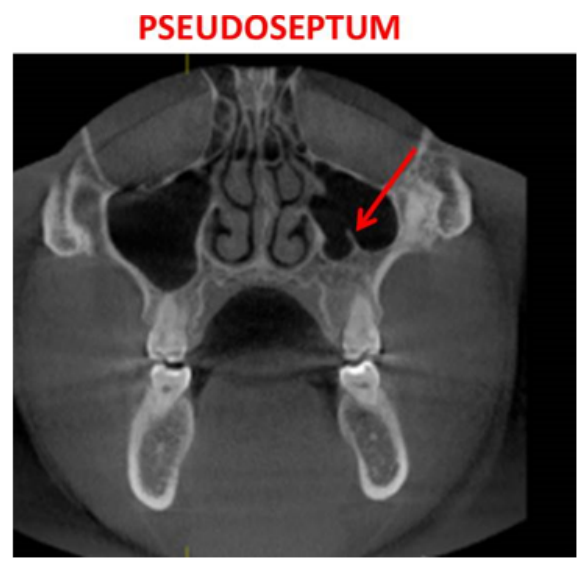

Fig. 2. Pseudoseptum de seno maxilar

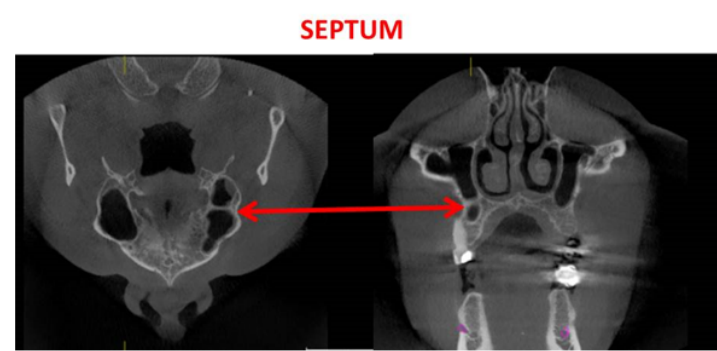

Fig. 3. Septum de seno maxilar

presente investigación se limitó a evaluar la frecuencia de hallazgos de variantes anatómicas del SM mediante TCCB, y en este punto coincide con el estudio de Shiki y colaboradores, hallando en primer término a la neumatización como la variante más frecuente.

Las variaciones en el seno maxilar por lo general son hallazgos de imagen, como se plantea en varios estudios realizados, por lo cual numerosos autores coinciden en la necesidad de realizar TCCB pre y post operatorias y así disminuir los posibles riesgos y complicaciones durante las cirugías. En el artículo de Rahpeyma A y Khajehahmadi $\mathbf{S}^{10}$ se habla de la importancia de la TCCB preoperatoria en los pacientes pre protésicos que desean aumentar la calidad y la cantidad de hueso para una adecuada rehabilitación oral. Se incluyeron 59 artículos en esta revisión; en donde se describieron y establecieron 10 parámetros que deben ser tenidos en cuenta y evaluados en las TCCB antes de realizar la apertura y el levantamiento del seno maxilar, esos parámetros fueron: el alto y el ancho de la cresta alveolar residual, el espesor de la pared lateral del seno maxilar, la presencia de la arteria antral alveolar y su diámetro, el ancho, angulación e irregularidad del suelo del seno maxilar, la relación íntima de la membrana de Schneider con las raíces de los dientes adyacentes, el septum del seno frontal, y la calidad del hueso subantral. Después de la evaluación se concluyó que, todos y cada uno de estos parámetros van a tener un alto impacto en el resultado de la cirugía del seno maxilar y un alto riesgo de fracaso si no son evaluados previamente. Al igual que los investigadores mencionados, el piloto realizado demuestra que un porcentaje mayoritario de pacientes son portadores de variantes anatómicas que podrían tener una influencia negativa en los diferentes procedimientos que se plantean ante las distintas necesidades de tratamiento de los pacientes, por esto, es de primordial interés el conocimiento y la correcta identificación de las mismas.

Dobele I y colaboradores ${ }^{11}$ realizaron una investigación con el objetivo de evaluar la presencia de variaciones anatómicas y patologías del seno maxilar utilizando una TCCB. Hallaron que había un engrosamiento de la mucosa en el $48,5 \%$ de los senos, 20,6\% presentaban tabiques y hubo opacidad en el 2,9\% de los senos. El orificio de drenaje del seno maxilar estuvo bloqueado en el $26,5 \%$ de las exploraciones y hubo una gran correlación entre los signos radiológicos de obstrucción del orificio del seno y la mucosa engrosada. Las variaciones anatómicas y lesiones del seno maxilar fueron hallazgos muy comunes en las TCCB. Por lo cual se sugiere que las tomografías se deben realizar como exámenes de rutina antes de la realización de cualquier tipo de procedimiento o tratamiento, ya que estas permiten una adecuada visualización del seno maxilar y ayuda a minimizar los riesgos antes, durante y después de la cirugía. El estudio piloto realizado coincide con la investigación de Dobele y colaboradores, dando como resultado que la mayoría de las TCCB analizadas muestran la presencia de variantes anatómicas.

En el año 2012, Lana y colaboradores ${ }^{12}$ realizaron un estudio que tuvo como objetivo evaluar las variantes anatómicas y las lesiones de los senos maxilares mediante el uso de TCCB en pacientes a los que se les estaba planificando un tratamiento de implantes en el maxilar superior. Dos radiólogos evaluaron 500 TCCB y donde encontraron que las variaciones anatómicas encontradas fueron neumatización $(83.2 \%)$, septo antral (44.4\%), hipoplasia (4.8\%) y exostosis $(2.6 \%)$. Este estudio concluye que encontrar lesiones y variaciones en los senos maxilares con TCCB es muy común y que es importante conocerlas ya que algunas de estas condiciones pueden modificar los tratamientos planificados y pueden requerir tratamientos especializados, conocer estas variaciones es importante en la práctica odontológica, sobre todo si se planea realizar implantes. El piloto examinó 40 TCCB (80 SM) de las cuales se encontró en un $68.8 \%$ de variantes anatómicas, siendo la neumatización la más frecuente con un $41.8 \%$; estando de acuerdo con los resultados de la investigación de los autores mencionados.

En el estudio realizado por Shahidi y colaboradores ${ }^{13}$ el propósito fue determinar las variaciones del seno maxilar mediante la ayuda de TCCB en población del sur de Irán. 
. Las variaciones anatómicas fueron evaluadas en una vista axial e incluyeron la presencia de neumatización alveolar o neumatización anterior, exostosis e hipoplasia, además de la localización y la altura de los septos sinusales y la localización de la arteria alveolar postero superior. Se examinaron en total 396 senos maxilares y se halló que la neumatización alveolar fue la variación anatómica más comúnmente detectada, la neumatización anterior fue detectada en 96 senos (24.2\%), septo antral fue hallado en 180 senos (45.4\%) y se localizó en más frecuentemente en la región anterior. La arteria alveolar postero superior fue hallada intraósea en 242 senos $(65.7 \%)$. Se llega a la conclusión de que las variaciones anatómicas de los senos maxilares se pueden hallar fácilmente mediante el uso de TCCB y que estas son de gran ayuda para la realización de un diagnóstico, así como para el tratamiento. Nuevamente los resultados del estudio piloto coinciden con los de la evidencia científica, dando a la neumatización de los SM como la variante anatómica más frecuente.

En el estudio realizado por Orhan y colaboradores ${ }^{14}$ el objetivo fue determinar la prevalencia, altura, localización y morfología de los septos del seno maxilar en pacientes dentados, parcialmente dentados y edéntulos adultos, así como niños en dentición decidua usando tomografía computarizada cone beam programados para intervenciones quirúrgicas. Se evaluaron 554 lados en 272 pacientes, en donde 30 fueron niños y 242 fueron adultos. La prevalencia localización y morfología fue evaluada en los 3 planos. La altura de los septos fue medida con el ángulo entre la dirección del septo y la sutura media palatina, las diferencias entre edades, localización y medidas fueron analizadas. Ellos encontraron que la prevalencia de segmentos del seno maxilar con septos fue del $58 \% .13(3.2 \%)$ septos en pacientes completamente edentulos, $198(53.9 \%)$ septos en pacientes dentados y 14 (3.8\%) septos en dentición decidua. La ubicación de los septos observada en todos los grupos de estudio demostró una prevalencia mayor $(69,1 \%)$ en la región media que en la parte anterior o posterior. No se observaron diferencias estadísticamente significativas en relación con el género o la edad, para la altura del tabique. Se encontró que los septos del seno maxilar son más altos en pacientes parcialmente desdentados que en los desdentados. En el estudio piloto realizado, a diferencia de la investigación de Orhan, la frecuencia de septos y pseudoseptos fue baja; presentándose 3 casos de septos $(5.4 \%)$ y 12 casos de pseudoseptos $(21.8 \%)$.

Por todo lo considerado, es necesaria una evaluación completa y detallada de estas estructuras anatómicas ante la necesidad de ejecutar tratamientos que las involucren. Las tecnologías actuales de imagen, en particular la TCCB aporta de manera significativa al cumplimiento de este objetivo, sugiriéndose un examen de este tipo previo a la elaboración del plan de tratamiento.

Dentro de las limitaciones del estudio, se pueden men-
- Al final el número de variables fue muy extenso, por el cruce y la posibilidad de encontrar las 5 variables en un solo paciente, esto retraso la calibración y dificulto un poco la recolección de la información. - Algunas tomografías no abarcaban el área total de interés.

\section{CONCLUSIÓN}

La frecuencia de variantes anatómicas presente en los senos maxilares evaluados con tomografía computarizada cone beam es alta.

Conflicto de intereses y financiamiento Los autores declaran no tener conflicto de intereses, haber cumplido con los requisitos de autoría y haber autofinanciado este artículo.

\section{Referencias}

1 Richard L. Wayne A. Gray's anatomy for students. 2da ed. España. Editorial ELSEVIER; 2006.

2 Testut L. Tratado de Anatomía Humana. $7^{\circ}$ ed. Barcelona: Editorial Salvat; 1925.

3 Méndez I, Vasallo V, Cenjor C. Embriología y anatomía de la nariz y de los senos paranasales. Variaciones anatómicas de las fosas nasales. Libro virtual de formación en otorrinolaringología. Cap; 41. (Consultado el 25 de junio de 2016) Disponible en http://seorl.net/libro-virtual/.

4 Ritter L, Lutz J, Neugebauer J, Scheer M, Dreiseidler T, Zinser MJ. Prevalence of pathologic findings in the maxillary sinus in cone-beam computerized tomography. Oral Surg Oral Med Oral Pathol Oral Radiol Endod. 2011; 111:634-40.

5 Graccoa A, Parentib S, Ioelec C, Bonettib A, Stellinid G. Prevalence of incidental maxillary sinus findings in Italian orthodontic patients: a retrospective cone-beam computed tomography study. Korean J Orthod. 2012; 42 (6): 329-34.

6 Prabhat M, Rai S, Kaur M, Prabhat K, Bhatnagar P, Panjwani S. Computed tomography based forensic gender determination by measuring the size and volume of maxillary sinuses. J Forensic Dent Sci. 2016; 8 (1): 40-6.

7 Eloy P. Nollevaux M.Bertrand B. Fisiología de los senos paranasales. EMC (Elsevier SAS), Otorrinolaringología. 2005; 20: 1-9.

8 Sánchez- Pérez A, Boracchia AC, López-Jornet P, BoixGarcía P. Characterization of the Maxillary Sinus Using Coen Beam Computed Tomography. A retrospective radiographic study. Implant Dent. 2016; 25 (6): 762-9. cionar: 
9 Shiki K, Tanaka T, Oda M, Kito S, Wakasugi-Sato N, Recibido: 5 de enero de 2017. Matsumoto-Takeda S, Nishimura $\mathrm{S}$ et al. The significance of cone beam computed tomography for the visualization of anatomical variations and lesions in the maxillary sinus for patients hoping to have dental implant-supported maxillary restorations in a private dental office in Japan. Head \& Face Medicine. 2014; 28: 10-20.

10 Rahpeyma A, Khajehahmadi S. Open Sinus Lift Surgery and the Importance of Preoperative Cone-Beam Computed Tomography Scan: A Review. JIOH. 2015; 7(9):127-33.

11 Dobele I, Kise L, Apse P, Kragis G, Bigestans A. Radiographic assessment of findings in the maxillary sinus using cone-beam computed tomography. Stomatologia 2013; 15: 119-22.

12 . Lana P. Carneiro M. De Souza E. Manzi R, Horta C. Anatomic variations and lesions of the maxillary sinus detected in cone beam computed tomography for dental implants. Clin Oral Implants Res. 2012; 23:1398- 1403.

13 . Shahidi S. Zamiri B. Danaei S. Salehi S. Hamedani S. Evaluation of Anatomic Variations in Maxillary Sinus with the Aid of Cone Beam Computed Tomography (CBCT) in a Population in South of Iran. J Dent Shiraz Univ Med Sci. 2016; 17(1): 7-15.

14 Orhan K. Kusakci B. Aksoy S. Bayindir H. Berberoğlu A. Seker E. Cone Beam CT Evaluation of Maxillary Sinus Septa Prevalence, Height, Location and Morphology in Children and an Adult Population. Med Princ Pract. 2013; 22: 47-53. 